\title{
First-Principle Wannier function analysis of the electronic structure of PdTe: Weaker magnetism and superconductivity
}

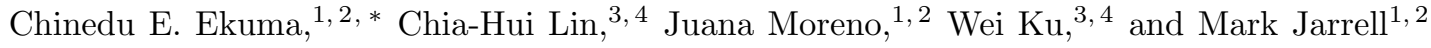 \\ ${ }^{1}$ Department of Physics 83 Astronomy Louisiana State University, Baton Rouge, LA 70803, USA \\ ${ }^{2}$ Center for Computation and Technology, Louisiana State University, Baton Rouge, LA 70803, USA \\ ${ }^{3}$ Department of Physics and Astronomy, Stony Brook University, Stony Brook, New York 11794, USA \\ ${ }^{4}$ Condensed Matter Physics and Materials Science Department, \\ Brookhaven National Laboratory, Upton, New York 11973, USA
}

(Dated: September 23, 2018)

\begin{abstract}
We report a first-principles Wannier function study of the electronic structure of PdTe. Its electronic structure is found to be a broad three-dimensional Fermi surface with highly reduced correlations effects. In addition, the higher filling of the $\mathrm{Pd} d$-shell, its stronger covalency resulting from the closer energy of the $\mathrm{Pd}-d$ and Te- $p$ shells, and the larger crystal field effects of the $\mathrm{Pd}$ ion due to its near octahedral coordination all serve to weaken significantly electronic correlations in the particle-hole (spin, charge, and orbital) channel. In comparison to the Fe Chalcogenide e.g., FeSe, we highlight the essential features (quasi-two-dimensionality, proximity to half-filling, weaker covalency, and higher orbital degeneracy) of Fe-based high-temperature superconductors.

PACS numbers: 31.15.A-, 74.70.Ad, 31.15.V-, 71.15.Ap,71.20.-b, 71.27.+a
\end{abstract}

\section{INTRODUCTION}

The discovery of high-temperature superconductivity in the $\mathrm{Fe}$ chalcogenides $1 / 2$ led to a "gold rush" to find superconductivity in other non-cuprate materials. Compared with Fe-based superconductors,,$\frac{311}{11}$ these non-toxic layered compounds with weak interlayer van der Waals forces exhibit interesting physical properties, including phase separation, $\frac{12}{12}$ strong correlations, $\frac{13 / 14}{11}$ non-trivial isovalent doping,$\frac{15}{17} \mathrm{Fe}$ excess effects, 18 Fe vacancy, 19 and rich high-pressure phase diagrams 13/1422 24 Attention has also shifted to other non-Fe transition metal based chalcogenides as they are shedding light on the road to the post-iron age in superconductivity.

There are yet no rigorous computational study of the electronic structure of Palladium monotelluride (PdTe). So far, experimental studies show that it has a $T_{c} \approx 2.3$ $-4.5 \mathrm{~K}, 25 \sqrt{28}$, electron-phonon coupling constant $\lambda_{e-p} \sim$ $1.4, \frac{25}{25}$ and a phase diagram reminiscent of the highpressure diagram of $\mathrm{Fe}$ chalcogenides $(\mathrm{FeCh}) \stackrel{23229}{\mathrm{Com}-}$ pared with $\mathrm{FeCh}{ }^{1 / 2|15| 24 \mid 30}$ the hexagonal PdTe structure (c.f. Fig. I( a)) can be regarded as the deformed structure of FeTe (c.f. Fig. II(c)) obtained by sliding the anion and cation layers. Despite such structural similarity, the local ligand field is transformed from a tetrahedral cage surrounding $\mathrm{Fe}$ to an octahedral cage surrounding Pd. With a similar crystal structure but distinct physical properties, PdTe thus serves as a good candidate for comparison to better illuminate the important physical effects and to reveal the building blocks for a stronger superconductivity in the Fe chalcogenides.

Motivated by the growing effort to understand properties of NiAs-like systems due to their very diverse and interesting properties, we provide a first-principles Wannier function analysis of the electronic structure of PdTe. We remark that there is no yet 'rigorous' electronic structure study of the properties of PdTe. A concomitant motivation is the evolving effort to understand the superconductivity mechanism in Fe-based superconductors. By comparing the characteristics of PdTe with those of $\mathrm{Fe}$ chalcogenides, one expects to identify key ingredients behind the high-temperature superconductivity in Fe-based superconductors, a current research topic of general interest to a broad audience.

We find that the face-shared octahedral coordination of $\mathrm{Pd}$ and the larger size of its $4 d$ orbital favor the electronic kinetic energy over the Coulomb potential energy. Also, a broad three-dimensional Fermi surface (FS) with a strong variation along the $k_{z}$ direction, and a lack of orbital degeneracy are observed. The almost fully occupied $\mathrm{Pd} d$ orbitals lead to a strong covalent $\mathrm{Pd}-\mathrm{Te}$ bonding and a vanishing local magnetic moment. A near octahedral coordination in PdTe leads to a large crystal field splitting of the Pd- $d$ orbitals $\left(t_{2 g}-e_{g}\right)$ with a crystal field parameter of $\Delta_{\text {oct }} \sim 400 \mathrm{meV}$. Therefore, most probably electronic correlations are suppressed in pure PdTe. One can thus conclude that due to these dilute correlations, pure PdTe may just be a low $T_{c}$ superconductor in basic agreement with the experimental work of Karki et al:25. This study illuminates the important underlying physics of the high-Tc Fe-based superconductors and most especially, reveals the building blocks for a stronger superconductivity in Fe chalcogenides. Such a study can help to reassess the rules one uses in the search of a room temperature superconductor and in particular, providing key insights on how to achieve high temperature superconductivity in general.

\section{APPROACH AND CRYSTAL STRUCTURE}

We use first-principles calculations to study the electronic structure of $\mathrm{PdTe}$ and the competition be- 


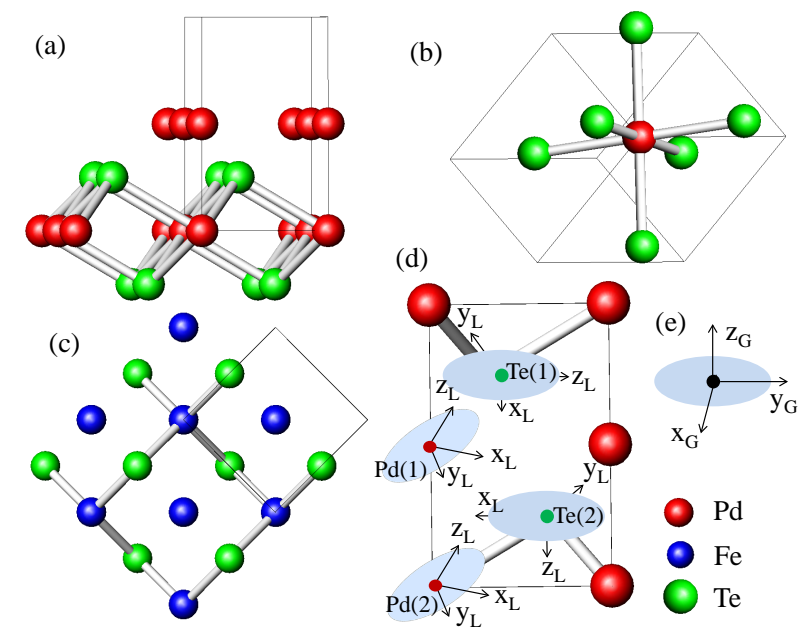

FIG. 1: (Color online) (a) The hexagonal structure of PdTe along the [001] direction. The positions of the atoms are $\operatorname{Pd}(1):(0,0,1 / 2), \operatorname{Pd}(2):(0,0,0), \operatorname{Te}(1):(1 / 3,2 / 3 / 3 / 4)$, and $\mathrm{Te}(2)$ : $(2 / 3,1 / 3,1 / 4)$, respectively. (b) The Pd-centered edge and face sharing environment with the neighboring octahedra. There are six Te octahedrally arranged around Pd. (c) The tetragonal structure of FeTe along the [001] direction. (d) The local coordinate of $\mathrm{Pd}$ and $\mathrm{Te}$ in the PdTe system as utilized in the definition of the Wannier basis. The local coordinates corresponding to each of the atoms in the unit cell (two $\mathrm{Pd}$ and two Te) have been defined using the x-convention. (e) The global coordinate of the PdTe system. The subscripts L and $\mathrm{G}$ denote local and global coordinates, respectively.

tween various magnetic configurations. We utilize standard density functional theory (DFT) within the general potential linearized augmented planewave (LAPW) method ${ }^{31}$ and the generalized gradient approximation (PBE-GGA) functional, ${ }^{32}$ as implemented in WiEN2k ${ }^{33}$ In our computations, we utilize the room temperature experimental lattice parameters: $\mathrm{a}=\mathrm{b}=4.152 \AA$ and $\mathrm{c}$ $=5.672 \AA \stackrel{34}{, 3}$ and the hexagonal crystal structure with space group $\mathrm{P} 6_{3} / \mathrm{mmc}$ (Patterson symbol). ${ }^{35} \mathrm{Pd}$ and Te atoms occupy $2 a$ and $2 c$ Wyckoff positions, respectively, namely, $\operatorname{Pd}(1):(0,0,1 / 2), \operatorname{Pd}(2):(0,0,0) ; \operatorname{Te}(1)$ : $(1 / 3,2 / 3 / 3 / 4)$, and $\operatorname{Te}(2):(2 / 3,1 / 3,1 / 4) .28 / 35 / 36$ Thus, the first-principles ground state can be reached with all the WiEN2k default settings and a k-mesh of $14 \times 14 \times 9$. To downfold the DFT electronic band structure, symmetry respecting Wannier functions $37 \sqrt[39]{39}$ of $\mathrm{Pd} d$ and Te $p$ are constructed to capture the low-energy Hilbert space within $[-8,3] \mathrm{eV}$ and obtain an effective tight-binding Hamiltonian. We then obtain the band structure and the Fermi surface by calculating the orbital-resolved spectral function, $A_{n, k}(\omega)$, where $n, k, \omega$ are orbital index, crystal momentum, and energy, respectively.

The structure of PdTe is tied strongly to its electronic and magnetic properties, since the spatial extension of the $4 d$ orbitals promotes comparable and competing kinetic and Coulomb energies. PdTe crystallizes in the NiAs or $\mathrm{D}_{6 h}^{4}$ crystal structure, which is of interest both from experimental and theoretical points of view $\underline{40 / 41}$ Transition metal compounds with the NiAs crystal structure attract special interest due to anomalies in their magnetic, elastic, and electrical properties, especially near phase transitions, the nature of which is still under study ${ }^{41}$ In PdTe the Pd atoms sit in an fcc-like environment, while the Te atoms form an hcp-like structure and are surrounded by six $\mathrm{Pd}$ metal atoms forming a trigonal prism ${ }^{42}$ In this structure, the Pd-centered octahedron shares both edges and faces with the neighboring $\mathrm{PdTe}_{6}$ octahedra (c.f. Fig. I (a) and (b)). This is different from $\mathrm{FeCh}$ or $\mathrm{FePn}$ where the corrugated square planes of $\mathrm{Fe}$ with Se (or As) atoms are such that Fe is tetrahedrally coordinated below and above the planes. Thus, the Fe atoms sit in the tetrahedral cavities of the tetragonally distorted close packed lattice of Se (or As) ${ }^{43}$ We also note that the structure of PdTe places Pd atoms closer to each other than in a perovskite; as such, direct PdPd hybridization becomes important as we will discuss below.

\section{RESULTS AND DISCUSSION}

Our resulting electronic band structure and density of states (DOS) for non-magnetic PdTe are shown in Fig. 2, colored to emphasize the $\mathrm{Pd} t_{2 g}, e_{g}$ and Te $p$ orbitals. The whole spectrum is predominantly a single huge band complex due to the strong hybridization between $\mathrm{Pd} d$ and Te $p$ orbitals. The role of $p-d$ hybridization in NiAstype compounds have been highlighted in literature (See for e.g., Refs. 4446 for discussions). Notice from the DOS (c.f. Fig. 2 ) that almost all the $\mathrm{Pd} d$ orbitals are occupied, and the low energy excitations near the Fermi surface are composed mainly of Te $p$ electrons hybridized with the $e_{g}$ orbital of Pd. The Pd $d$ orbital occupancy can be calculated from the trace of the one-particle reduced density matrix on each $\mathrm{Pd}$ atom (in Wannier function basis) and is found to be 9.31 , which is very close to the fully occupied value of 10 . This indicates strong covalency of the Pd-Te bonding and strong suppression of the spin local moment. The strong covalency can also be confirmed by measuring the distance $\Delta E_{p-d}$ between the highest peaks in Te $5 p$ and $\mathrm{Pd} 4 d$ bands which is a measure of the ionicity or covalency of the bonding. $\Delta E_{p-d}$ is found to be $\approx 2.92$ a.u. We find in particular that the electronic structure of PdTe is determined by short-range interactions in the Te $p-\operatorname{Pd} d$ band complex, with the ligand-field splitting of the $\mathrm{Pd} d$ states in the environment of the Te atoms being the key determinant of the structure of the $d$ band.

In Fig. 3, the Fermi surface is presented in various $k_{z}$ planes. As already seen in the band structure, the states in the proximity of the Fermi level consist mainly of Te $p$ (green orbital) weakly hybridized with $\mathrm{Pd} e_{g}$ (red orbital), thus appearing dark green. The Fermi energy is located close to the local maxima of the DOS, corresponding to $\mathrm{N}\left(\mathrm{E}_{\mathrm{F}}\right) \approx 2.41$ a.u. The corresponding elec- 


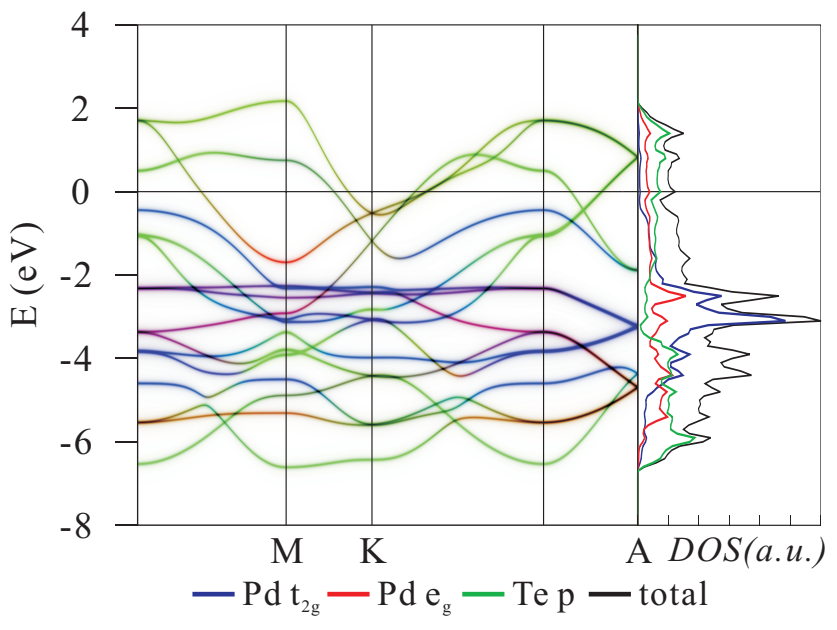

FIG. 2: (Color online) Left panel: Calculated dispersion (band structure) of PdTe within [-8.0, 3.0] eV. Right panel: The corresponding spectra (in a.u. unit) as obtained from the symmetry respecting Wannier functions within [-8.0, 3.0] eV. In both plots, the Fermi level is set equal to zero.

tronic specific heat coefficient $\gamma$ is $2.84 \mathrm{~mJ} \mathrm{~mol}{ }^{-1} \mathrm{~K}^{-2}$. This value is in good agreement with the experimental value of $6.0 \mathrm{~mJ} \mathrm{~mol}^{-1} \mathrm{~K}^{-2}$ obtained by Karki et al 25 if we take into account the many-body effect contributions from electron-phonon interactions such that $\gamma$ is renormalized as $\gamma_{n}=\gamma\left(1+\lambda_{e-p}\right)$.

To investigate the magnetism of PdTe, we survey various magnetic configurations, including collinear, bicollinear, and checkerboard antiferromagnetism, with spin-polarized calculations. Indeed, the non-polarized case possesses the lowest ground state total energy, so there are no long-range magnetic correlations. This is consistent with the fact that the almost-full-Pd- $d$ orbitals not only rule out the superexchange mechanism for antiferromagnetic correlations, but also suppress the effectiveness of Hund's coupling, and thus ferromagnetic correlations as well.

In order to gain a microscopic insight into the electronic structure of PdTe, we transform the self-consistent Kohn-Sham (DFT) Hamiltonian to Wannier basis, as summarized in Table I. In the intra-atomic Pd block, one finds a large $(\sim 400 \mathrm{meV}) t_{2 g}-e_{g}$ splitting which originated from the octahedral ligand field, with negligible offdiagonal terms associated with the tiny distortion of the octahedral cage. A similar splitting $(\sim 500 \mathrm{meV})$ is also found between Te $p_{z}$ and $p_{x} / p_{y}$. Furthermore, we find considerable inter-atomic $\mathrm{Pd}-\mathrm{Te}$ and $\mathrm{Pd}-\mathrm{Pd}$ hopping, for example, $t_{P d(1)_{z^{2}}, T e(1)_{p_{x}}}=509, t_{P d(1)_{z^{2}}, T e(1)_{p_{y}}}=881$, $t_{P d(1)_{x^{2}-y^{2}}, T e(2)_{p_{x}}}=-881, t_{P d(1)_{z^{2}}, T e(1)_{p_{z}}}=559$, and $t_{P d(1)_{x y}, P d(2)_{d_{x y}}}=-353 \mathrm{meV}$, which are much larger than the intrinsic scale for the on-site energy difference between $\mathrm{Pd}$ and $\mathrm{Te}(\sim 100 \mathrm{meV})$ (c.f. TableI). The same hopping value of $t_{P d(1)_{z^{2}}, T e(1)_{p_{y}}}$ and $t_{P d(1)_{x^{2}-y^{2}}, T e(2)_{p_{x}}}$,

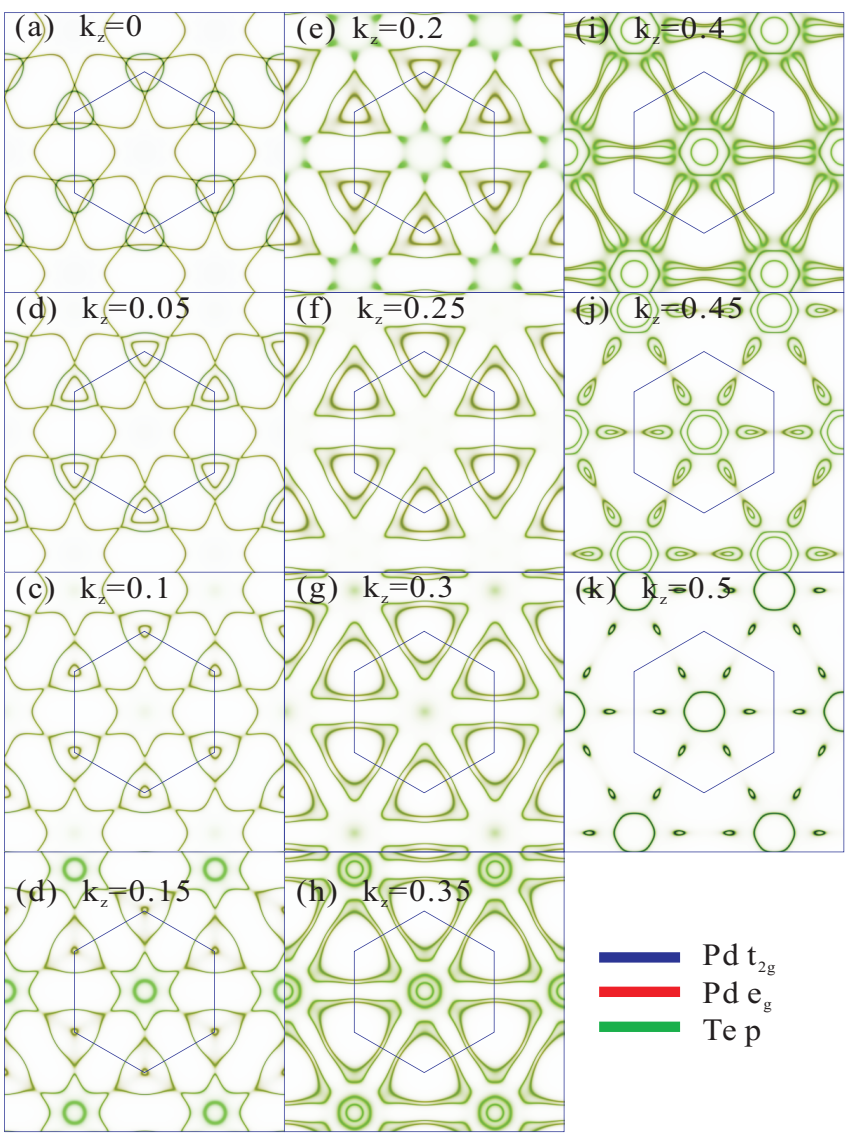

FIG. 3: (Color online) The Fermi surface of PdTe at different $k_{z}$ planes with the same color code as Fig. 2 The value of $k_{z}$ is in units of $2 \pi / c$. The states in the proximity of the Fermi surface are predominantly Te- $p$ states, hence the color of the Fermi surface plots is mainly green.

even when they are not symmetry related, is due to the $30^{\circ}$ tilting angle that is naturally built-in in the crystal symmetry of PdTe, as can be understood from the SlaterKoster coefficients used in the tight-binding model. $47 / 48$

Unconventional superconductivity generally emerges in frustrated systems $\$ 993$ when long-range order in the spin, charge or orbital channel is suppressed. The key ingredients needed for a typical unconventional superconductor, strong short-range correlations in these channels, are either negligible or dilute in PdTe.

Several factors make PdTe very different from $\mathrm{FeCh}$. Strong hopping due to the more extended $4 d$ orbitals, which promotes a larger kinetic energy, closer $\mathrm{Pd}-\mathrm{Pd}$ distance in the face-shared $\mathrm{Pd}$ octahedron environment, negligible Hund's moment, weaker magnetic spin fluctuations, lack of orbital degeneracy, strong covalency, and very dilute magnetic frustration. Although PdTe has a layered structure similar to the tetragonal Fe chalcogenides, its intrinsic $\mathrm{Pd} 4 d$ character and face-shared octahedron environment not only suppress the electronic correlations in the charge, orbital, and spin channels but 
TABLE I: (Color online). This table is mainly intended to show the hopping dominated physics in PdTe and, as such, it represents a reduced matrix of the original 16 by 16 matrix of $\mathrm{Pd} d$ and Te $p$ states. For clarity, the $\operatorname{Pd}(1) d$ and $\mathrm{Te}(1) p$ sub-bands are represented using bold letters. The expectation value $\left\langle\mathrm{WF}_{\mathrm{i}}|\mathrm{H}| \mathrm{WF}_{\mathrm{j}}\right\rangle$, where $\mathrm{WF}_{\mathrm{i}}$ corresponds to the Wannier function of orbital $i$, is denoted as $\left\langle\mathrm{n}|\mathrm{H}| \mathrm{n}^{\prime}\right\rangle$ on the table. Onsite energies of $\mathrm{Pd} d$ orbitals, split due to crystal fields into $\mathrm{e}_{\mathrm{g}}\left(z^{2}\right.$ and $\left.\mathrm{x}^{2}-\mathrm{y}^{2}\right)$ (red) and $\mathrm{t}_{2 \mathrm{~g}}(\mathrm{yz}, \mathrm{xz}$, and $\mathrm{xy}$ ) (blue) subbands, and the Te $p_{x}, p_{y}$, and $p_{z}$ sub-bands (green), and the hopping integrals among $\mathrm{Pd} d$ and Te $p$ Wannier orbitals for the nonmagnetic case are displayed. Units are meV. The local coordinates of each of the atoms are defined in Fig. I(d).

\begin{tabular}{lccccc|ccc}
\hline \hline$\left\langle\mathrm{n}|\mathrm{H}| \mathrm{n}^{\prime}\right\rangle$ & $\mathbf{z}^{\mathbf{2}}$ & $\mathbf{x}^{\mathbf{2}}-\mathbf{y}^{\mathbf{2}}$ & $\mathbf{y z}$ & $\mathbf{x z}$ & $\mathbf{x y}$ & $\mathbf{x}$ & $\mathbf{y}$ & $\mathbf{z}$ \\
\hline $\mathbf{z}^{\mathbf{2}}$ & -2608 & 0 & 2 & 2 & 4 & 509 & 881 & 559 \\
$\mathbf{x}^{\mathbf{2}}-\mathbf{y}^{\mathbf{2}}$ & 0 & -2608 & -4 & 4 & 0 & 38 & -22 & 0 \\
$\mathbf{y z}$ & 2 & -4 & -2987 & -42 & 42 & 187 & -290 & 361 \\
$\mathbf{x z}$ & 2 & 4 & -42 & -2987 & 42 & -345 & 17 & 361 \\
$\mathbf{x y}$ & 4 & 0 & 42 & 42 & -2987 & 26 & 45 & 33 \\
\hline $\mathbf{x}$ & 509 & 38 & 187 & -345 & 26 & -3112 & 0 & 0 \\
$\mathbf{y}$ & 881 & -22 & -290 & 17 & 45 & 0 & -3112 & 0 \\
$\mathbf{z}$ & 559 & 0 & 361 & 361 & 33 & 0 & 0 & -2597 \\
\hline \hline$z^{2}$ & -20 & 0 & 60 & 60 & 120 & 8 & 14 & 50 \\
$x^{2}-y^{2}$ & 0 & 20 & 104 & -104 & 0 & -22 & 13 & 0 \\
$y z$ & 60 & 104 & -150 & -353 & 150 & 2 & 0 & -33 \\
$x z$ & 60 & -104 & -353 & -150 & 150 & -1 & 1 & -33 \\
$x y$ & 120 & 0 & 150 & 150 & -353 & 18 & 31 & -1 \\
\hline$x$ & 509 & -881 & 52 & 158 & -158 & 79 & 254 & 263 \\
$y$ & -38 & -22 & 0 & 307 & 307 & 254 & -214 & -455 \\
$z$ & 279 & -484 & 33 & -361 & 361 & -263 & 455 & 524 \\
\hline \hline
\end{tabular}

also promote three dimensionality. The three dimensionality of PdTe electronic structure is made clear from the strong $k_{z}$ variation of the Fermi surface topology in Fig. 3. Such a strong three-dimensionality indicates a much weaker Fermi surface nesting, and would likely favor a weaker long-range correlation in the particle-hole (spin, charge, and orbital) channel. In addition, since the charge-transfer energy between $\mathrm{Pd} d$ and chalcogen $p$ states is much smaller in PdTe than in FeCh, the $\mathrm{Pd} d$ states are almost fully occupied, and there are no states available to form a large Hund's moment, making magnetic correlations and, e.g., magnetic frustration effects, weaker. Therefore, any possibility of magnetically-driven superconductor is suppressed. The relatively low superconducting temperature in PdTe may likely be due to another source, presumably phonons.

As can be seen from Table [ the dominant hoppings are those between $\mathrm{Pd}$ and the neighboring Te. This strong hopping is due to the large orbital overlap between Te and Pd ions (c.f. Fig. I(d)). On the other hand, a strong $\mathrm{Pd}-\mathrm{Pd}$ hopping is also observed. This is due to the spatial extension of the $4 d$ orbitals, a consequence of its larger quantum number compared to $3 d$ orbitals, that overlap with neighboring $\mathrm{Pd}$ ions; and the closer $\mathrm{Pd}-\mathrm{Pd}$ distance in the octahedral environment. Since the hybridization matrix between the $d$-orbitals scales as $\mathrm{d}^{-4}$, where $\mathrm{d}$ is the bond length, a shorter bond length greatly affects the hybridization between $d$-orbitals. ${ }^{[57}$ This will increase the kinetic energy, compared to the case of corner-shared and edge-shared octahedrons. Hence, the importance of correlations from direct Coulomb interactions will be highly dilute. Also, the lack of orbital degeneracy will eliminate the ferro-orbital correlation and the associated C-type antiferromagnetic order 58

The strong covalency, absence of electronic correlations, lack of orbital degeneracy, and the dilute nature of the magnetic frustration in PdTe are in sharp contrast, in particular, to Fe chalcogenides, and in general, to Fe-based superconductors. Thus, PdTe will display dramatically weaker magnetic and superconducting tendencies than the Fe-based superconductors. One may conclude that the key ingredients for a high $T_{c}$ unconventional superconductivity are lost and pure PdTe may just be a low $T_{c}$ superconductor. However, interesting magnetic correlation might be reactivated upon Fe doping.

\section{SUMMARY}

We have performed self-consistent DFT and downfolding electronic band structure via symmetry respecting Wannier functions to study the electronic properties of PdTe. Our computations show that there is significant $\mathrm{Pd}-d$ and Te- $p$ hybridization, larger crystal field splitting of Pd d-orbitals due to their near octahedral coordination, and higher filling of the $\mathrm{Pd} d$-shell resulting in weaker magnetic frustration, strong covalency, and lack of orbital degeneracy, which quench ferro-orbital correlations. The large $k_{z}$ variation of the Fermi surface topology also demonstrates a strong three-dimensional character of the electronic structure. These features destroy the ingredients needed for PdTe to be a high- $\mathrm{T}_{\mathrm{c}}$ unconventional superconductor. This case study provides a good contrast that highlights some important features (quasitwo-dimensionality, proximity to half-filling, weaker covalency, and higher orbital degeneracy) of Fe-based hightemperature superconductors.

\section{Acknowledgments}

We thank Carol Duran for carefully reading the manuscript. Work at LSU is funded by the National Science Foundation LA-SiGMA award: EPS 1003897. Work at BNL is supported by the U.S. Department of Energy (DOE) under contract DE-AC02-98CH10886. Interinstitutional collaboration is supported by the DOECMCSN grant DE-AC02-98CH10886. High performance computational resources are provided by the Louisiana 
* corresponding email: cekuma1@lsu.edu

1 F.-C. Hsu, J.-Y. Luo, K.-W. Yeh, T.-W. Huang, P. M. Wu, Y.-C. Lee, D. C. Yan, and M.-K. Wu, Proc. Natl. Acad. Sci. U.S.A. 85, 14262 (2008).

2 T. Ozaki, Y. Mizuguchi, S. Demura, K. Deguchi, Y. Kawasaki, T. Watanabe, H. Okazaki, H. Hara, H. Takeya, and T. Yamaguchi, J. Appl. Phys. 104, 013912 (2012).

3 H. A. Mook, M. D. Lumsden, A. D. Christianson, S. E. Nagler, B. C. Sales, R. Jin, M. A. McGuire, A. S. Sefat, D. Mandrus, T. Egami, et al., Phys. Rev. Lett. 104, 187002 (2010).

4 Y. Kamihara, T. Watanabe, M. Hirano, and H. Hosono, J. Am. Chem. Soc. 104, 3296 (2008).

${ }^{5}$ C. Wang, L. Li, S. Chi, Z. Zhu, Z. Ren, Y. Li, Y. Wang, X. Lin, Y. Luo, S. Jiang, et al., Euro. Phys. Lett. 104, 67006 (2008).

6 M. Norman, Physics 1, 21 (2008).

7 M. Rotter, M. Tegel, and D. Johrendt, Phys. Rev. Lett. 101, 107006 (2008).

8 V. Zinth, V. Petricek, M. Dusek, and D. Johrendt, Phys. Rev. B 85, 014109 (2012).

${ }^{9}$ D. R. Parker, M. J. Pitcher, P. J. Baker, I. Franke, T. Lancaster, S. J. Blundell, and S. J. Clarke, Chem Comm. 1, 2189 (2009).

10 S. Deng, J. Köhler, and A. Simon, Phys. Rev. B 80, 214508 (2009).

11 M. Klanjšek, P. Jeglič, B. Lv, A. M. Guloy, C. W. Chu, and D. Arčon, Phys. Rev. B 84, 054528 (2011).

12 H. Hu, J.-M. Zuo, J. Wen, Z. Xu, Z. Lin, Q. Li, G. Gu, W. K. Park, and L. H. Greene, New J. Phys. 13 (2011).

13 S. Chi, J. A. Rodriguez-Rivera, J. W. Lynn, C. Zhang, D. Phelan, D. K. Singh, R. Paul, and P. Dai, Phys. Rev. B 84, 214407 (2011).

14 J. Wen, G. Xu, G. Gu, J. M. Tranquada, and R. J. Birgeneau, Reports on Progress in Physics 74, 124503 (2011).

15 M. H. Fang, H. M. Pham, B. Qian, T. J. Liu, E. K. Vehstedt, Y. Liu, L. Spinu, and Z. Q. Mao, Phys. Rev. B 78, 224503 (2008).

16 D. J. Gawryluk, J. Fink-Finowicki, A. Wiśniewski, R. Puźniak, R. Domukhovski, D. Diduszko, M. Kozlowski, and M. Berkowski, Supercond. Sci. Technol. 24, 065011 (2011).

17 Y. F. Nie, D. Telesca, J. I. Budnick, B. Sinkovic, and B. O. Wells, Phys. Rev. B 82, 020508 (2010).

18 Sudesh, S. Rani, S. Das, R. Rawat, C. Bernhard, and G. D. Varma, Journal of Applied Physics 111, 07 E119 (2012).

19 T. Berlijn, P. J. Hirschfeld, and W. Ku, arXiv:1204.2849v1.

${ }^{20}$ F. Chen, M. Xu, Q. Q. Ge, Y. Zhang, Z. R. Ye, L. X. Yang, J. Jiang, B. P. Xie, R. C. Che, M. Zhang, et al., Phys. Rev. X 1, 021020 (2011).

21 Z. Wang, Y. J. Song, H. L. Shi, Z. W. Wang, Z. Chen, H. F. Tian, G. F. Chen, J. G. Guo, H. X. Yang, and J. Q. Li, Phys. Rev. B 83, 140505 (2011).

${ }^{22}$ Y. Mizuguchi, F. Tomioka, S. Tsuda, T. Yamaguchi, and Y. Takano, Appl. Phys. Lett. 93, 152505 (2008).

23 Y. Mizuguchi, F. Tomioka, S. Tsuda, T. Yamaguchi, and Y. Takano, Physica C: Superconductivity 469, 1027
(2009)

24 S. Margadonna, Y. Takabayashi, Y. Ohishi, Y. Mizuguchi, Y. Takano, T. Kagayama, T. Nakagawa, M. Takata, and K. Prassides, Phys. Rev. B 80, 064506 (2009).

25 A. B. Karki, D. A. Browne, S. Stadler, J. Li, and R. Jin, J. Phys.: Condens. Matter 24, 055701 (2012).

26 B. T. Matthias, Phys. Rev. 92, 874 (1953).

27 B. T. Matthias, Phys. Rev. 90, 487 (1953).

28 A. Kjekshus and W. B. Pearson, Can. J. Phys. 43, 438-449 (1965).

29 L. Malavasi and S. Margadonna, Chem. Soc. Rev. 41, 3897 (2012).

30 A. Martinelli, A. Palenzona, M. Tropeano, C. Ferdeghini, M. Putti, M. R. Cimberle, T. D. Nguyen, M. Affronte, and C. Ritter, Phys. Rev. B 81, 094115 (2010).

31 D. J. Singh, Planewaves, Pseudopotentials, and the LAPW Method, 2nd Ed. (Springer-Velag, Berlin, 2006).

32 J. P. Perdew, K. Burke, and M. Ernzerhof, Phys. Rev. Lett. 77, 3865 (1996).

${ }^{33}$ P. Blaha, K. Schwarz, G. Madsen, D. Kvasnicka, and J. Luitz, WIEN2K, An Augmented Plane Wave+Local Orbitals Program for Calculating Crystal Structure (K. Schwarz Technical University, Wien, Austria, 2001).

${ }^{34}$ F. Gronvold and E. Rost, Acta Chemica Scandinavica 10 (1956).

35 T. Hahn, (ed.), International Tables for Crystallography, vol. A: Space-Group Symmetry (Springer, 2005).

36 E. Fluck, J. Res. Natl. Inst. Stand. Technol. 101, 217 (1996).

37 W. Ku, H. Rosner, W. E. Pickett, and R. T. Scalettar, Phys. Rev. Lett. 89, 167204 (2002).

38 W. Ku, T. Berlijn, and C.-C. Lee, Phys. Rev. Lett. 104, 216401 (2010).

39 T. Berlijn, D. Volja, and W. Ku, Phys. Rev. Lett. 106, 077005 (2011).

40 K. Bärner, phys. stat. sol. (a) 88, 13 (1978).

41 L. M. Sandratskii, R. F. Egorov, and A. A. Berdyshev, phys. stat. sol. (b) 103, 511 (1981).

42 M. Grundmann, The Physics of Semiconductors: An Introduction Including Devices and Nanophysics (Springer, 2006), 1st ed.

43 D. J. Singh, Physica C 469, 418 (2009).

${ }^{44}$ L. F. Mattheiss, Phys. Rev. B 10, 995 (1974).

45 S. R. Krishnakumar, N. Shanthi, P. Mahadevan, and D. D. Sarma, Phys. Rev. B 61, 16370 (2000).

46 H. W. Myron and F. M. Mueller, Phys. Rev. B 17, 1828 (1978).

47 J. C. Slater and G. F. Koster, Phys. Rev. 94, 1498 (1954).

48 W. A. Harrison, Electronic Structure and Properties of Solids (Dover, New York, 1989).

49 H. B. Yang, Z. Wang, and H. Ding, Phys.: Condens. Matter 19, 355004 (2007).

50 C. Piefke, L. Boehnke, A. Georges, and F. Lechermann, Phys. Rev. B 82, 165118 (2010).

51 C. Lacroix, P. Mendels, and F. Mila, Introduction to Frustrated Magnetism: Materials, Experiments, Theory, Springer Series in Solid-State Sciences (Springer, 2011).

${ }^{52}$ D. J. Scalapino, J. Low Temp. Phys. 117, 179 (1999). 
53 A. J. Drew, F. L. Pratt, T. Lancaster, S. J. Blundell, P. J. Baker, R. H. Liu, G. Wu, X. H. Chen, I. Watanabe, V. K. Malik, et al., Phys. Rev. Lett. 101, 097010 (2008).

${ }^{54}$ P. Vilmercati, A. Fedorov, I. Vobornik, U. Manju, G. Panaccione, A. Goldoni, A. S. Sefat, M. A. McGuire, B. C. Sales, R. Jin, et al., Phys. Rev. B 79, 220503 (2009).

${ }^{55}$ T. Yoshida, I. Nishi, S. Ideta, A. Fujimori, M. Kubota, K. Ono, S. Kasahara, T. Shibauchi, T. Terashima, Y. Mat- suda, et al., Phys. Rev. Lett. 106, 117001 (2011).

56 M. Fang, J. Yang, F. F. Balakirev, Y. Kohama, J. Singleton, B. Qian, Z. Q. Mao, H. Wang, and H. Q. Yuan, Phys. Rev. B 81, 020509 (2010).

57 G.-M. Zhao, Phys. Rev. B 62, 11639 (2000).

58 C.-C. Lee, W.-G. Yin, and W. Ku, Phys. Rev. Lett. 103, 267001 (2009). 